\title{
Development of a Recombinase Polymerase Amplification Assay for the Detection of Pathogenic Leptospira
}

\author{
Ahmed Ahmed *, Hans van der Linden and Rudy A. Hartskeerl \\ KIT Biomedical Research, WHO/FAO/OIE and National Leptospirosis Reference Centre, \\ Royal Tropical Institute, Meibergdreef 39, Amsterdam 1105, The Netherlands; \\ E-Mails: H.v.d.Linden@ kit.nl (H.L.); r.hartskeerl@kit.nl (R.A.H.) \\ * Author to whom correspondence should be addressed; E-Mail: a.ahmed@kit.nl; \\ Tel.: +31-205-662-317; Fax: +31-206-971-841.
}

Received: 21 February 2014; in revised form: 8 April 2014 / Accepted: 30 April 2014 /

Published: 8 May 2014

\begin{abstract}
Detection of leptospires based on DNA amplification techniques is essential for the early diagnosis of leptospirosis when anti-Leptospira antibodies are below the detection limit of most serological tests. In middle and low income countries where leptospirosis is endemic, routine implementation of real-time PCR is financially and technically challenging due to the requirement of expensive thermocycler equipment. In this study we report the development and evaluation of a novel isothermal recombinase polymerase amplification assay (RPA) for detection of pathogenic Leptospira based on TwistAmp chemistry. RPA enabled the detection of less than two genome copies per reaction. Retrospective evaluation revealed a high diagnostic accuracy (sensitivity and specificity of $94.7 \%$ and $97.7 \%$, respectively) compared to culturing as the reference standard. RPA presents a powerful tool for the early diagnosis of leptospirosis in humans and in animals. Furthermore, it enables the detection of the causative agent in reservoirs and environment, and as such is a valuable adjunct to current tools for surveillance and early outbreak warning.
\end{abstract}

Keywords: recombinase; polymerase; diagnosis; amplification; pathogenic; Leptospira; development; diagnostic; evaluation; RPA 


\section{Introduction}

Leptospirosis is a zoonotic disease with an estimated global burden of 873,000 severe annual cases and 49,000 deaths [1]. The causative agent comprises pathogenic species of the genus Leptospira. This bacterium circulates worldwide. It tolerates different climates and environments and can adapt to a wide range of animal hosts. Because the clinical manifestations mimic a variety of febrile infectious illnesses such as rickettsioses, dengue, and other viral haemorrhagic infections, the impact of the disease in the area where these diseases are endemic is often underrated. Because of the difficult diagnosis of leptospirosis, adequate surveillance is mostly lacking. Hence, the understanding of the transmission modes and risks and, notably, the dynamics leading to outbreaks is still in its infancy. Adequate tools for early diagnosis enabling early outbreak warning are badly needed.

Culturing from blood collected at the early stage of disease provides evidence of a leptospiral infection. However, leptospires are tedious, slow growing bacteria and it takes weeks to months for a culture to become positive. Thus, culturing as a diagnostic tool is not beneficial for the treatment of the individual patient. Nor does serology contribute to early diagnosis of leptospirosis because anti-Leptospira antibodies only become detectable in the late acute phase, 3-5 days after the onset of the disease. Laboratory diagnosis of leptospirosis in the early acute phase of the disease relies on molecular methods, particularly DNA amplification techniques, on blood samples [2,3]. To date, few real-time polymerase chain reactions (PCRs) have been validated and are currently in use in various laboratories. However, implementation of this technique as a routine diagnostic tool for leptospirosis is technically and financially problematic in middle and low income countries where the disease is endemic. The technique requires sophisticated expensive thermocycler equipment, subjected to regular maintenance and availability of a stable power supply. For this reason, isothermal amplification techniques avoiding the use of expensive and complicated thermocyclers in addition to the possibility of reading results by eye have been propagated as simple and affordable alternative molecular diagnostic tools [4-8]. However, in practice, the application of isothermal approaches such as the loop-mediated isothermal amplification (LAMP) for detection of leptospires is very limited [9]. Moreover, its diagnostic sensitivity and specificity is disputable and further intensive optimization and validation of this method is required [1]. Recently recombinase polymerase amplification (RPA) has been developed as a simple and fast isothermal amplification technique using affordable equipment. In addition to a regular DNA polymerase, this method employs a recombinase enzyme, single-stranded DNA binding proteins and homologous oligonucleotides to invade within the double-stranded target DNA, hence permitting sequence specific priming of DNA polymerase reactions without prior denaturation of template DNA. Because of the use of regular enzymes, the reaction is performed at a moderate and constant temperature $\left(37-39^{\circ} \mathrm{C}\right)$. Moreover, the chemistry of this technique enables both real-time readout or end-point 'sandwich assays', such as lateral-flow (LF) strips [10] and thus allows application in a variety of settings, ranging from sophisticated ones to point of care situations. In this study, we developed and evaluated an RPA test for detection of pathogenic Leptospira, using the TwistAmp Exo probe detection system for real-time readout. 


\section{Materials and Methods}

\subsection{Ethics Statement}

Procedures for collecting patients' data and use of clinical specimens for laboratory service improvement falls under the umbrella of the 'National Coordination Infectious Disease Control' (Landelijke Coördinatie Infectieziektebestrijding, LCI) 'Centre for Infectious Disease Control' (Centrum Infectieziektebestrijding, Cib) [11] which is a formal body of the Netherlands Ministry of Health and resides in the National Institute for Public Health and Environment (RIVM) in Bilthoven, The Netherlands and thus were conducted in compliance with the regulation, policies and principles of the Dutch Public Health Service Policy. The procedure includes the processing of anonymous data from patients upon receipt of a written informed consent.

\subsection{Leptospira Strains and Others Microorganisms}

Forty strains belonging to pathogenic, non-pathogenic and intermediate Leptospira species [12-16] and five other micro-organisms (Table 1) were included in this study. Leptospira strains were from the collection of the WHO/FAO/OIE and National Leptospirosis Reference Centre (NRL) in Amsterdam, The Netherlands. Other micro-organisms or their genomic DNA were gifts from colleagues from the Department of Biomedical Research and from other institutions.

Table 1. Leptospira strains and other microorganism used in this study.

\begin{tabular}{|l|l|l|l|l|l|l|}
\hline No. & Species & Serovar & Strain & Status & Reference & Result \\
\hline 1 & L. alexanderi & Manzhuang & A23 & Pathogenic & {$[12]$} & + \\
2 & L. alexanderi & Manhao 3 & L 60 & Pathogenic & {$[12]$} & + \\
3 & L. biflexa & Andamana & CH 11 & Non-pathogenic & {$[12]$} & - \\
4 & L. biflexa & Patoc & Patoc I & Non-pathogenic & {$[12]$} & - \\
5 & L. borgpetersenii & Castellonis & Castellon 3 & Pathogenic & {$[12]$} & + \\
6 & L. borgpetersenii & Nyanza & Kibos & Pathogenic & {$[12]$} & + \\
7 & L. borgpetersenii & Hardjo-bovis & L550 & Pathogenic & {$[12]$} & + \\
8 & L. borgpetersenii & Ceylonica & Piyasena & Pathogenic & {$[12]$} & + \\
9 & L. borgpetersenii & Mini & Sari & Pathogenic & {$[12]$} & + \\
10 & L. borgpetersenii & Hardjo-Bovis & Sponselee & Pathogenic & {$[12]$} & + \\
11 & L. borgpetersenii & Ballum & Mus 127 & Pathogenic & {$[12]$} & + \\
12 & L. broomii & Not determined & 5399T & Intermediate & {$[13]$} & - \\
13 & L. fainei & Hurstbridge & BUT 6T & Intermediate & {$[15]$} & - \\
14 & L. interrogans & Australis & Ballico & Pathogenic & {$[12]$} & + \\
15 & L. interrogans & Djasiman & Djasiman & Pathogenic & {$[12]$} & + \\
16 & L. interrogans & Hardjo & Hardjoprajitno & Pathogenic & {$[12]$} & + \\
17 & L. interrogans & Canicola & Hond Utrecht IV & Pathogenic & {$[12]$} & + \\
18 & L. interrogans & Bratislava & Jez Bratislava & Pathogenic & {$[12]$} & + \\
19 & L. interrogans & Pomona & Pomona & Pathogenic & {$[12]$} & + \\
20 & L. interrogans & Copenhageni & Wijnberg & Pathogenic & {$[12]$} & + \\
\hline
\end{tabular}


Table 1. Cont.

\begin{tabular}{|c|c|c|c|c|c|c|}
\hline No. & Species & Serovar & Strain & Status & Reference & Result \\
\hline 21 & L. kirschneri & Tsaratsovo & B $81 / 7$ & Pathogenic & {$[12]$} & + \\
\hline 22 & L. kirschneri & Butembo & Butembo & Pathogenic & {$[12]$} & + \\
\hline 23 & L. kirschneri & Vanderhoedeni & Kipod 179 & Pathogenic & {$[12]$} & + \\
\hline 24 & L. kirschneri & Grippotyphosa & Moskva V & Pathogenic & {$[12]$} & + \\
\hline 25 & L. kirschneri & Cynopteri & $3522 \mathrm{C}$ & Pathogenic & {$[12]$} & + \\
\hline 26 & L. licerasiae & Varillal & VAR 010T & Intermediate & [14] & - \\
\hline 27 & L. weilii & Ranarum & ICF & Pathogenic & [16] & + \\
\hline 28 & L. meyeri & Semaranga & $\begin{array}{l}\text { Veldrat Semarang } \\
173\end{array}$ & Non-pathogenic & [12] & - \\
\hline 29 & L. noguchii & Nicaragua & 1011 & Pathogenic & {$[12]$} & + \\
\hline 30 & L. noguchii & Louisiana & LSU 1945 & Pathogenic & [12] & + \\
\hline 31 & L. noguchii & Argentiniensis & Peludo & Pathogenic & [12] & + \\
\hline 32 & L. noguchii & Proechimys & $1161 \mathrm{U}$ & Pathogenic & {$[12]$} & + \\
\hline 33 & L. santarosai & Balboa & $735 \mathrm{U}$ & Pathogenic & {$[12]$} & + \\
\hline 34 & L. santarosai & Rio & $\operatorname{Rr} 5$ & Pathogenic & [12] & + \\
\hline 35 & L. santarosai & Shermani & $1342 \mathrm{~K}$ & Pathogenic & {$[12]$} & + \\
\hline 36 & L. vanthielii & Holland & WaZHolland & Non-pathogenic & {$[12]$} & - \\
\hline 37 & L. weilii & Coxi & Cox & Pathogenic & {$[12]$} & + \\
\hline 38 & L. weilii & Celledoni & Celledoni & Pathogenic & {$[12]$} & + \\
\hline 39 & L. wolbachi & Codice & $\mathrm{CDC}$ & Non-pathogenic & {$[12]$} & - \\
\hline 40 & L. yanagawae & Saopaulo & Sao Paulo & Non-pathogenic & {$[12]$} & - \\
\hline 41 & $\begin{array}{l}\text { Acinetobacter } \\
\text { calcoaceticus }\end{array}$ & & Other microorganism & & & - \\
\hline 42 & Bacillus subtilis & & Other microorganism & & & - \\
\hline 43 & Borrelia burgdorferi & & Other microorganism & & & - \\
\hline 44 & Leptonema illini & & Other microorganism & & & - \\
\hline 45 & Treponema pallidum & & Other microorganism & & & - \\
\hline
\end{tabular}

\subsection{Clinical Samples}

This retrospective evaluation was executed on 63 clinical samples (59 serum and 4 EDTA blood) submitted to the NRL for confirmation of suspected leptospirosis All clinical specimens were collected from patients at 1 to 10 days post onset of disease (DPO). The study sample consisted of 19 samples that yielded a positive culture and 44 samples that were negative by culture. From all 19 patients who had a positive culture, leptospirosis was also confirmed by serology on paired samples, consistent with our case definition [17]. From eight of the 44 cases that scored as culture negatives, a follow up sample was received that showed negative results in the serodiagnosis. All specimens were anonymized and randomized prior to testing. Results of other diagnostic tests were unknown to the tester performing the RPA. 


\subsection{DNA Extractions}

Leptospires were propagated in EMJH liquid media at $30{ }^{\circ} \mathrm{C}$ as described by Ellinghausen and McCullough [18] as modified by Johnson and Harris [19]. The concentration of bacteria was determined by counting in a Helber bacteria chamber (Weber Scientific international, West Sussex, UK) according to the standard protocol. All genomic DNAs from leptospires and other micro-organisms in culture medium and from $200 \mu \mathrm{L}$ serum or blood were extracted, purified and eluted in $0.1 \times \mathrm{TE}$ buffer pH 8.0 by using the QIAamp DNA extraction kit (Qiagen, Hilden, Germany) or by using MagNA Pure Systems according to the manufacturer's instructions (Roche Diagnostics, Mannheim, Germany). The quantity of Leptospira genomic DNA was estimated by measuring absorbance of DNA using the Spectrophotometer ND-1000 Nanodrop (Wilmington, DE, USA). Leptospira interrogans serovar Copenhageni, strain Wijnberg was used as the basic strain for the development, optimization and initial evaluation of the RPA. Based on the published genome of Copenhageni, one genome equivalent corresponds to $5.1 \times 10^{-15}$ g DNA [20].

\subsection{Primers and Probes Design}

Both probe (exoProK1) and forward and reverse primers (exoPriFK1 and exoPriRK2, respectively), for the recombinase polymerase amplification assay (RPA) specifically detecting pathogenic Leptospira were deduced from a conserved partial sequence of the gene lipL32 from Leptospira interrogans serovar Copenhageni strain Fiocruz L1-130, GenBank: AE016823.1, sequence 1666423 to 1666512 (90 bp) as part of this study (Figure 1). A complete matching of these primers and probe to homologous sequence of pathogenic Leptospira was established by in silico analysis using Basic Local Alignment Search Tool (BLAST) [21]. The probe was labelled according to TwistAmp exo Probe chemistry with a fluorophore (FAM) and a Black Hole Quencher-1 (BHQ1) at its 3' end and a tetrahydrofuran (THF) spacer in between (dTFAM-THF-dTQuencher).

Figure 1. Partial lipL32 gene sequence used as RPA amplification target. Presentation of the RPA 90 nucleotides sized amplification locus and positions of the forward (exoPriFK1) and reversed (exoPriRK2) primers and the probe (exoProK1) used in the reaction. Nucleotides are indicated by small letters. Capitals in the probe indicate the following: $\mathrm{F}=$ Fluorophore (FAM), H = Tetrahydrofuran (THF) spacer, $\mathrm{Q}=$ Black Hole Quencher-1.

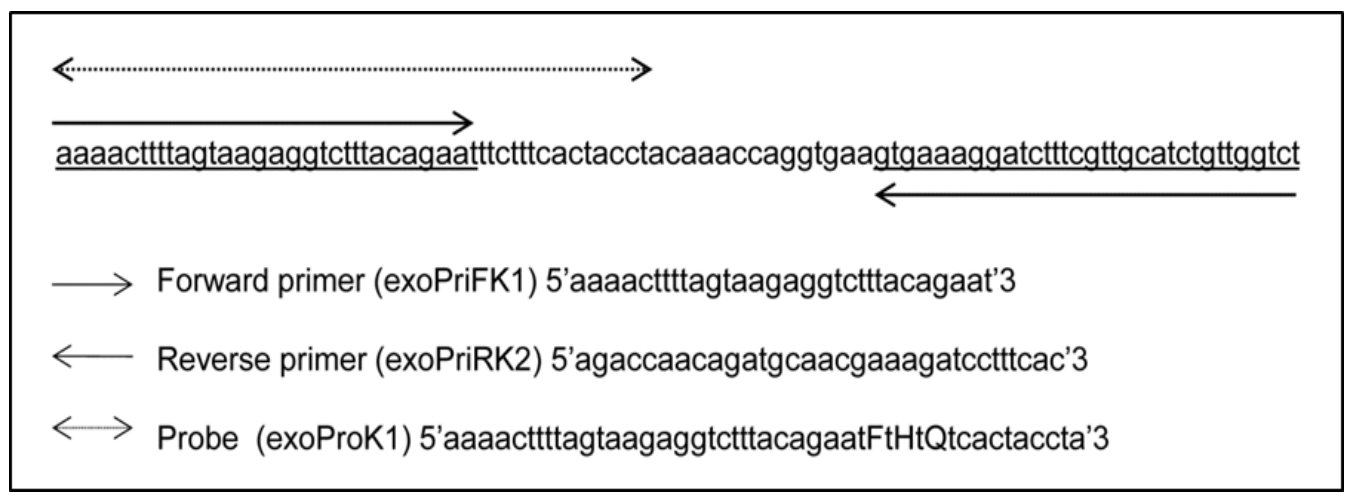




\subsection{Recombinase Polymerase Amplification: Reagents and Conditions}

To perform the RPA, the TwistAmp exo kit (TwistDX, Cambridge, UK) was used. All the reagents with the exception of the rehydration buffer, magnesium acetate and the positive reaction control were included in the kit as freeze-dried pellet. The primers and the probe were added to the mix after dissolving the pellet in the $1 \mathrm{x}$ rehydration buffer followed by adding the target DNA template. Varying concentrations of primers, probe, magnesium acetate at different reaction temperatures varying between 37 and $41{ }^{\circ} \mathrm{C}$, which present critical parameters, were used to select optimal RPA conditions. For optimization, the total volume of the reaction was $50 \mu \mathrm{L}$ containing 1000 copies from Leptospira interrogans strain Wijnberg as DNA template. $10 \mu \mathrm{L}$ sterile water was used instead of DNA template as a negative control. The positive control included in the kit was used to check the activity of the reagents and the performance of the reaction as recommended by the manufacturer.

The test was performed on CFX96 real-time PCR detection system (Bio-Rad). In case of an optimal amplification, the machine was programmed at constant temperature $\left(38{ }^{\circ} \mathrm{C}\right)$ as follows: (i) $38{ }^{\circ} \mathrm{C}$ for $4 \mathrm{~min}$ as incubation period followed by mixing and a spin down step (ii) then incubation at $38^{\circ} \mathrm{C}$ for $2 \mathrm{~s}$ followed by (iii) fluorescence detection. The last two steps (ii) and (iii) were repeated 120 times. The total reaction time was 25 minutes.

\subsection{Determination of Analytical and Diagnostic Sensitivity and Specificity}

The analytical sensitivity and the performance of the assay was monitored as described previously [20], using genomic DNA isolated from L. interrogans serovar Copenhageni strain Wijnberg. Briefly, 10-fold serial dilutions of genomic DNA were used as template to perform the test and to construct an amplification standard curve. The last 10-fold dilutions still giving a positive signal were subjected to subsequent 2-fold serial dilutions. We set the end-point at the dilution in which the assay detects the target in at least $95 \%$ of the replicates. The amplification performance of the RPA was compared with that of two previously published real-time PCRs, namely SYBR green PCR targeting $\sec Y$ [20] and TaqMan PCR targeting lipL32 [22], both performed as described by the authors. The analytical specificity was examined using 45 Leptospira strains and other microorganism as listed in Table 1. The diagnostic sensitivity and specificity and the confidence intervals were calculated using the 63 clinical samples according to standard literature [23-25]. All samples used in this study were tested in duplicate.

\section{Results}

\subsection{Optimal Reaction Conditions and RPA Analytical Sensitivity and Specificity}

A reaction temperature of $38^{\circ} \mathrm{C}$ and concentrations of $420 \mathrm{nM}$ for each the primer, $60 \mathrm{nM}$ for the probe and $16.8 \mathrm{mM}$ magnesium acetate were found to provide an optimal performance of the RPA. Applying this condition, appropriate exponential amplification was achieved and the detection limit of the assay (LOD) was estimated at $\leq 10 \mathrm{fg}$ or $\leq 2$ genome equivalents based on the standard curve constructed from serial dilutions of genomic DNA. This compares well with the LOD of the two real-time PCRs included in the evaluation, which also allowed detection of about two genome copies 
(results not shown). RPA gave positive results for all pathogenic Leptospira, and a negative result for all saprophytic and intermediate species as well as for all other pathogenic microorganisms included in the evaluation, consistent with the specificity of the test for pathogenic Leptospira spp. as deduced from in silico analysis (Table 1).

\subsection{The Diagnostic Sensitivity and Specificity}

63 clinical samples were enrolled in this study to calculate the diagnostic sensitivity and specificity. Based on results from culturing as the reference test, RPA had diagnostic sensitivity (DSe) and specificity (DSp) of $94.7 \%$ (95\% CI, 71.9 to 99.7\%) and 97.7\% (95\% CI, 86.5 to 99.9\%), respectively. Among 19 positive culture patient samples, one gave negative result when tested with RPA assay. In contrast, one culture negative sample gave a positive result in RPA.

\section{Discussion}

In this study, we report for the first time the development and evaluation of an RPA assay for the detection of pathogenic Leptospira [10]. The gene lipL32 was used as the amplification target for RPA. lipL32 codes for a major lipoprotein located in the outer membrane. The gene is confined to pathogenic Leptospira spp. and its overall sequence is well conserved [26]. A high analytical specificity was obtained by selecting an amplification locus of 90 nucleotides within this gene for deducing primers and a probe that shared $100 \%$ sequence homology with all available sequences from strains of pathogenic Leptospira spp. The in silico specificity of the assay was confirmed by application of RPA on a panel of bacteria, including saprophytic and intermediate Leptospira spp., confirming that amplification was restricted to pathogenic leptospiral strains. These pathogenic strains included strain ICF that previously has been re-positioned from the saprophytic species, L. meyeri, to the pathogenic Leptospira clade. [12,16,20,27-33], once again confirming its pathogenic status.

In this study the analytical sensitivity was estimated based on counting the number of the bacteria in culture prior to DNA extraction and a subsequent determination of the concentration of the DNA by spectrophotometry. The detection limit, established at $<2$ genome copies, compared well with that of two validated real-time PCRs, one based on SYBR Green technology and the other one on TaqMan chemistry [20,22]. This substantiates a similar technical performance of this much simpler RPA compared to the sophisticated PCR formats.

To determine the diagnostic accuracy of RPA, culturing was used as the reference test. Culturing provides proof of infection and previous reports suggest that culturing as a reference test is superior to the standard microscopic agglutination test (MAT) [17,34]. Our evaluation revealed a very satisfying level of diagnostic accuracy for RPA with a DSe and DSp of 94.7\% and 97.7\%, respectively. One sample, consisting of EDTA blood gave a positive result by RPA however it was negative by culturing. From this patient, no follow-up sample, required for serological confirmation, was obtained. However, the diagnostic SYBR Green PCR on the single sample gave an indeterminate outcome, suggesting that leptospiral DNA might have been present in the sample at a low concentration. Furthermore, a positive RPA associated with a negative culture result can well be explained both by stochastic effects as well as by taking into account that EDTA is deleterious to the viability of leptospires [35]. The possibility of false positive result by carryover contamination is not obvious, 
since this format of RPA reaction is performed in a closed system that evades the risk of cross-over. A stochastic cause is also a plausible explanation for the one sample that was negative by RPA and positive by culture. The culture was found to belong to a pathogenic species L. interrogans serovar Hebdomadis (data not shown). Thus, the possibility of a negative RPA result because of an infection with an intermediate species is excluded. This is not surprisingly as isolation of leptospires belonging to intermediate species generally is associated with asymptomatic infection or mild disease [13-15]. Additionally, the retrospective nature of this study implies that RPA has been executed on samples that have been stored for months to years, not always under conditions that optimally preserve the integrity of the leptospiral DNA. RPA amplifies a much shorter DNA segment than is usually targeted by PCR. Hence, it is less susceptible to detrimental effects on the quality and quantity of the amplification target during storage. However, damage of the target DNA leading to a reduced apparent diagnostic sensitivity cannot be fully ruled out in this evaluation. Indeed, this specific sample had been stored for 2 years and a consequent false negative outcome of RPA presents a likely explanation. This is further substantiated by the fact that leptospirosis was also confirmed by serology on a paired sample using the standard microscopic agglutination test.

RPA with a real-time readout offers marked advantages above other previously described amplification assays for Leptospira detection, including isothermal ones. The technique is more rapid than any other amplification method, generating a result within $25 \mathrm{~min}$ and the reaction is performed at an ambient constant temperature. Consequently, it evades the need for an expensive and sophisticated thermocycler. Basically, it only requires a simple battery driven handheld fluorometer in point-of-care and field setting.

RPA is less sensitive to inhibitors than PCR [10] and is capable of amplifying DNA from a variety of samples from humans, animals and the environment. The test allows multiplexing and thus has a potential use for differential diagnosis of several clinically similar diseases [36] or allows detailing of the infecting Leptospira at the species and even strain level. These are important features for adequate surveillance. In principle, this real-time RPA approach generates digital data that can be linked to the Global System for Mobile Communications (GSM) network and, hence, might be helpful in creating diagnostic data clouds, accessible through internet by various stakeholders (clinicians, patients, epidemiology sections at national reference centers and/or Ministries of Health and Agriculture) through dedicated passwords. Simplification is possible by using the endpoint amplification read-out through the lateral flow platform [10], which shows a promising but not yet optimal performance (data not shown). The lateral flow presents a point-of-care device, which can be labelled with a Quick Response $(\mathrm{QR})$ code linked to clinically and epidemiologically relevant data. The image of the test result and code could then be transmitted by SMART phone. Alternatively, sophisticated but more reliable lab-on-a-chip approaches are possible, for example by supplying the device with a microchip that becomes active by hybridization to the detection strip. Thus RPA presents a potentially powerful and innovative tool for surveillance and early outbreak detection that could contribute to a better understanding and prediction of outbreaks. Our study shows that RPA may present a highly valuable and attractive alternative to early diagnosis by currently available molecular amplification methods. However, our study has been performed retrospectively and comprised a relative small sample of cases, hence affecting the statistical validity of the data. Since leptospirosis is a relatively rare disease in the Netherlands, a prospective study including statistically relevant numbers will take several years 
of execution. To overcome this drawback, we aim at a further prospective evaluation of the diagnostic accuracy of this RPA in settings where leptospirosis is more prevalent.

\section{Conclusions}

In this study for the first time, we describe and evaluate an RPA assay for the detection of pathogenic Leptospira in clinical samples. The analytical and diagnostic sensitivity and specificity of the assay were satisfactory. RPA is an isothermal reaction which can be performed with simple equipment therefore RPA is particularly suitable in point-of-care and field setting. The method is very fast, less sensitive to amplification inhibitors and enables both real-time readout or end-point detection. Our test has capability to detect the pathogen in reservoir animals and in a variety of environment samples. Hence, the Leptospira RPA could contribute to the effort toward better understanding the dynamic of the infection during outbreaks.

\section{Author Contributions}

Conceived and designed the experiments: Ahmed Ahmed; Rudy A. Hartskeerl. Performed the experiments: Ahmed Ahmed; Hans van der Linden. Analyzed the data: Ahmed Ahmed; Rudy A. Hartskeerl. Contributed reagents/materials/analysis tools: Rudy A. Hartskeerl. Wrote the paper: Ahmed Ahmed; Rudy A. Hartskeerl.

\section{Conflicts of Interest}

The authors declare no conflict of interest.

\section{References}

1. Picardeau, M.; Bertherat, E.; Jancloes, M.; Skouloudis, A.N.; Durski, K.; Hartskeerl, R.A. Rapid tests for diagnosis of leptospirosis: Current tools and emerging technologies. Diagn. Microbiol. Infect. Dis. 2014, 78, 1-8.

2. Ahmed, A.; Engelberts, M.F.; Boer, K.R.; Ahmed, N.; Hartskeerl, R.A. Development and validation of a real-time PCR for detection of pathogenic Leptospira species in clinical materials. PLoS One 2009, 4, doi:10.1371/journal.pone.0007093.

3. Levett, P.N. Leptospirosis. Clin. Microbiol. Rev. 2001, 14, 296-326.

4. Colebrander, A.; Terpstra, W.J.; Hartskeerl, R.A. Detectie van pathogene leptospiren met behulp van NASBA. Ned Tijdschr Geneeskd. 1994, 138, 436.

5. Koizumi, N.; Nakajima, C.; Harunari, T.; Tanikawa, T.; Tokiwa, T.; Uchimura, E.; Furuya, T.; Mingala, C.N.; Villanueva, M.A.; Ohnishi, M.; Suzuki, Y. A new loop-mediated isothermal amplification method for rapid, simple, and sensitive detection of Leptospira spp. in urine. J. Clin. Microbiol. 2012, 50, 2072-2074.

6. Lin, X.; Chen, Y.; Lu, Y.; Yan, J.; Yan, J. Application of a loop-mediated isothermal amplification method for the detection of pathogenic Leptospira. Diagn. Microbiol. Infect. Dis. 2009, 63, 237-242. 
7. Sonthayanon, P.; Chierakul, W.; Wuthiekanun, V.; Thaipadungpanit, J.; Kalambaheti, T.; Boonsilp, S.; Amornchai, P.; Smythe, L.D.; Limmathurotsakul, D.; Day, N.P.; Peacock, S.J. Accuracy of loop-mediated isothermal amplification for diagnosis of human leptospirosis in Thailand. Am. J. Trop. Med. Hyg. 2011, 84, 614-620.

8. Suwancharoen, D.; Kulchim, C.; Chirathaworn, C.; Yoshida, S. Development of a novel primer combination to detect pathogenic Leptospira by loop-mediated isothermal amplification. J. Microbiol. Methods 2012, 91, 171-173.

9. Ahmed, A.; Grobusch, M.P.; Klatser, P.R.; Hartskeerl, R.A. Molecular approaches in the detection and characterization of Leptospira. J. Bacteriol. Parasitol. 2012, 3, doi:10.4172/21559597.1000133.

10. Piepenburg, O.; Williams, C.H.; Stemple, D.L.; Armes, N.A. DNA detection using recombination proteins. PLoS Biol. 2006, 4, doi:10.1371/journal.pbio.0040204.

11. Hartskeerl, R.A. Leptospirosen. In LCI-richtlijnen Infectieziektebestrijding; Van Steenbergen, J.E., Timen, A., Beaujean, D.J.M.A., Eds.; Landelijke Coordinatie Infectieziektebestrijding: Bilthoven, The Netherland, 2008; pp. 238-243.

12. Brenner, D.J.; Kaufmann, A.F.; Sulzer, K.R.; Steigerwalt, A.G.; Rogers, F.C.; Weyant, R.S. Further determination of DNA relatedness between serogroups and serovars in the family Leptospiraceae with a proposal for Leptospira alexanderi sp. nov. and four new Leptospira genomospecies. Int. J. Syst. Bacteriol. 1999, 49, 839-858.

13. Levett, P.N.; Morey, R.E.; Galloway, R.L.; Steigerwalt, A.G. Leptospira broomii sp. nov., isolated from humans with leptospirosis. Int. J. Syst. Evol. Microbiol. 2006, 56, 671-673.

14. Matthias, M.A.; Ricaldi, J.N.; Cespedes, M.; Diaz, M.M.; Galloway, R.L.; Saito, M.; Steigerwalt, A.G.; Patra, K.P.; Ore, C.V.; Gotuzzo, E.; et al. Human leptospirosis caused by a new, antigenically unique Leptospira associated with a Rattus species reservoir in the Peruvian Amazon. PLoS Negl. Trop. Dis. 2008, 2, doi:10.1371/journal.pntd.0000213.

15. Perolat, P.; Chappel, R.J.; Adler, B.; Baranton, G.; Bulach, D.M.; Billinghurst, M.L.; Letocart, M.; Merien, F.; Serrano, M.S. Leptospira fainei sp. nov. isolated from pigs in Australia. Int. J. Syst. Bacteriol. 1998, 48, 851-858.

16. Victoria, B.; Ahmed, A.; Zuerner, R.L.; Ahmed, N.; Bulach, D.M.; Quinteiro, J.; Hartskeerl, R.A. Conservation of the $S 10-s p c-\alpha$ locus within otherwise highly plastic genomes provides phylogenetic insight into the genus Leptospira. PLoS One 2008, 3, doi:10.1371/journal.pone.0002752.

17. Goris, M.G.A.; Leeflang, M.M.; Boer, K.R.; Goeijenbier, M.; van Gorp, E.C.M.; Wagenaar, J.F.; Hartskeerl, R.A. Establishment of valid laboratory case definition for human leptospirosis. J. Bacteriol. Parasitol. 2012, 3, doi:10.4172/2155-9597.1000132.

18. Ellinghausen, H.C., Jr.; McCullough, W.G. Nutrition of Leptospira Pomona and growth of 13 other serotypes: Fractionation of oleic albumin complex and a medium of bovine albumin and polysorbate 80. Am. J. Vet. Res. 1965, 26, 45-51.

19. Johnson, R.C.; Harris, V.G. Differentiation of pathogenic and saprophytic letospires. I. Growth at low temperatures. J. Bacteriol. 1967, 94, 27-31. 
20. Ahmed, A.; Engelberts, M.F.; Boer, K.R.; Ahmed, N.; Hartskeerl, R.A. Development and validation of a real-time PCR for detection of pathogenic Leptospira species in clinical materials. PLoS One 2009, 4, 10.1371/journal.pone.0007093.

21. BLAST: Basic Local Alignment Search Tool. Available online: http://www.blast.ncbi.nlm.nih.gov/ Blast.cgi (accessed on 28 March 2013).

22. Stoddard, R.A.; Gee, J.E.; Wilkins, P.P.; McCaustland, K.; Hoffmaster, A.R. Detection of pathogenic Leptospira spp. through TaqMan polymerase chain reaction targeting the lipL32 gene. Diagn. Microbiol. Infect. Dis. 2009, 64, 247-255.

23. Newcombe, R.G. Two-sided confidence intervals for the single proportion: Comparison of seven methods. Stat. Med. 1998, 17, 857-872.

24. Wilson, E. Probable inference, the law of succession, and statistical inference. J. Am. Stat. Assoc. 1927, 22, 209-212.

25. VassarStats: Website for Statistical Computation. Available online: http://www.vassarstats.net (accessed on 12 December 2013).

26. Haake, D.A.; Chao, G.; Zuerner, R.L.; Barnett, J.K.; Barnett, D.; Mazel, M.; Matsunaga, J.; Levett, P.N.; Bolin, C.A. The leptospiral major outer membrane protein LipL32 is a lipoprotein expressed during mammalian infection. Infect. Immun. 2000, 68, 2276-2285.

27. Gravekamp, C.; van de Kemp, H.; Franzen, M.; Carrington, D.; Schoone, G.J.; Van Eys, G.J.; Everard, C.O.; Hartskeerl, R.A.; Terpstra, W.J. Detection of seven species of pathogenic leptospires by PCR using two sets of primers. J. Gen. Microbiol. 1993, 139, 1691-1700.

28. Hookey, J.V. Characterization of Leptospiraceae by $16 \mathrm{~S}$ DNA restriction fragment length polymorphisms. J. Gen. Microbiol. 1993, 139, 1681-1689.

29. Hookey, J.V.; Bryden, J.; Gatehouse, L. The use of $16 \mathrm{~S}$ rDNA sequence analysis to investigate the phylogeny of Leptospiraceae and related spirochaetes. J. Gen. Microbiol. 1993, 139, 2585-2590.

30. Letocart, M.; Baranton, G.; Perolat, P. Rapid identification of pathogenic Leptospira species (Leptospira interrogans, L. borgpetersenii, and L. kirschneri) with species-specific DNA probes produced by arbitrarily primed PCR. J. Clin. Microbiol. 1997, 35, 248-253.

31. Murgia, R.; Riquelme, N.; Baranton, G.; Cinco, M. Oligonucleotides specific for pathogenic and saprophytic Leptospira occurring in water. FEMS Microbiol. Lett. 1997, 148, $27-34$.

32. Segers, R.P.; van Gestel, J.A.; Van Eys, G.J.; van der Zeijst, B.A.; Gaastra, W. Presence of putative sphingomyelinase genes among members of the family Leptospiraceae. Infect. Immun. 1992, 60, 1707-1710.

33. Ahmed, A.; Anthony, R.M.; Hartskeerl, R.A. A simple and rapid molecular method for Leptospira species identification. Infect. Genet. Evol. 2010, 10, 955-962.

34. Limmathurotsakul, D.; Turner, E.L.; Wuthiekanun, V.; Thaipadungpanit, J.; Suputtamongkol, Y.; Chierakul, W.; Smythe, L.D.; Day, N.P.; Cooper, B.; Peacock, S.J. Fool's gold: Why imperfect reference tests are undermining the evaluation of novel diagnostics: A reevaluation of 5 diagnostic tests for leptospirosis. Clin. Infect. Dis. 2012, 55, 322-331.

35. Nervig, R.M.; Ellinghausen, H.C., Jr. Viability of Leptospira interrogans serotype Grippotyphosa in swine urine and blood. Cornell Vet. 1978, 68, 70-77. 
36. Word Health Organization. Human Leptospirosis: Guidance for Diagnosis, Surveillance and Control; WHO: Geneva, Switzerland, 2003.

(C) 2014 by the authors; licensee MDPI, Basel, Switzerland. This article is an open access article distributed under the terms and conditions of the Creative Commons Attribution license (http://creativecommons.org/licenses/by/3.0/). 